\title{
Le cerveau endocrine
}

La physiologie des régulations repose sur un concept fondamental introduit par Claude Bernard : le maintien de la constance du milieu intérieur, " condition d'une existence libre et indépendante ". La plupart des fonctions physiologiques concourent au maintien de cette constance du milieu intérieur - aussi appelée "homéostasie " - indispensable à la survie de l'organisme lorsqu'il est soumis à des variations plus ou moins importantes de l'environnement. La plupart des cellules sont isolées de l'environnement, mais sont en relation au sein de l'organisme par l'intermédiaire du milieu intérieur, représenté par l'ensemble des liquides extra-cellulaires (LEC). Pour réaliser ce maintien de l'homéostasie, deux systèmes de communication permettent à l'organisme une réaction adaptée : le système endocrinien, qui s'adresse potentiellement à toutes les cellules par l'intermédiaire des LEC ; le système nerveux, qui fonctionne en réseaux ordonnés stables et s'adresse aux seules cellules nerveuses.

Depuis un peu plus de soixante ans, l'idée d'un dialogue possible entre ces deux systèmes de communication, que l'on croyait auparavant indépendants, est apparue avec la mise en évidence du concept de neurosécrétion en 1928. Certains neurones hypothalamiques ont un double rôle : de neurone, cellule qui reçoit et transmet une information de type électrique, et de cellule endocrine, qui synthétise et libère une hormone dans les LEC. Ces neurones se comportent comme transducteurs neuroendocriniens (traduction d'une information nerveuse en une information endocrinienne). De ce concept est née peu à peu une discipline nouvelle, la neuroendocrinologie, qui étudie les influences réciproques qu'exercent l'un sur l'autre les systèmes nerveux et endocrinien, non seulement le contrôles des sécrétions endocrines par le cerveau (comme nous allons le voir plus précisément par l'intermédiaire $\mathrm{du}$ complexe hypothalamohypophysaire), mais aussi les modulations exercées par les hormones sur le fonctionnement du système nerveux.

C'est avec la description précise des rapports anatomiques et physiologiques entre l'hypophyse et le système nerveux central, plus particulièrement l'hypothalamus, que s'est véritablement développée la neuroendocrinologie.

L'hypothalamus constitue moins de $1 \%$ du volume total du cerveau ; et pourtant il contient des systèmes neuronaux à l'origine de la régulation d'un grand nombre de fonctions vitales concourant au maintien de l'homéostasie et donc à la survie de l'individu (prise de nourriture et de boisson, homéothermie...) mais aussi à la survie de l'espèce (reproduction). Situé à la base du cerveau, sur le plancher du troisième ventricule, l'hypothalamus s'étend du chiasma optique, en avant, aux corps mammillaires, en arrière ; il est grossièrement divisé en trois régions : l'hypothalamus périventriculaire, qui est au voisinage immédiat du troisième ventricule; l'hypothalamus latéral, constitué d'un ensemble de systèmes multisynaptiques ascendants et descendants; l'hypothalamus médian, qui contient la plupart des noyaux hypothalamiques $(\mathrm{NH})$ bien délimités parmi lesquels ceux qui sont directement impliqués dans la neurosécrétion endocrinienne. L'hypothalamus est prolongé à sa base par l'infundibulum ou tige pituitaire par lequel il est en relation avec l'hypophyse, ou glande pituitaire, logée dans la cavité osseuse de la selle turcique.

L'hypophyse est constituée de deux tissus glandulaires distincts : l'antéou adénohypophyse et la post- ou neurohypophyse. La compréhension des relations entre l'hypothalamus et ces deux parties de l'hypophyse fonctionnellement distinctes retrace l'histoire de la neuroendocrinologie.

La tige pituitaire et la neurohypophyse sont constituées par les axones et les terminaisons des neurones hypothalamiques magnocellulaires (noyaux supra-optiques et paraventriculaires) qui synthétisent et libèrent dans la circulation sanguine générale deux neurohormones peptidiques : l'hormone antidiurétique et l'ocytocine. L'ocytocine synchronise et stimule la contraction des fibres musculaires lisses de l'utérus, facilitant la parturition, ainsi que celles de la glande mammaire, facilitant l'éjection du lait; l'hormone antidiurétique agit au niveau du néphron rénal en favorisant la réabsorption de l'eau (on l'appelle également vasopressine du fait de ses propriétés hypertensives).

Les glandes endocrines périphériques (thyroïde, glandes surrénales, testicules, ovaires) sont stimulées par des hormones sécrétées par l'adénohypophyse. Un rôle essentiel est joué dans ce contrôle par un système circulatoire reliant l'hypothalamus et l'adénohypophyse : le système porte hypothalamo-hypophysaire (SPHH), situé au niveau de l'éminence médiane, dans le plancher de l'hypothalamus. C'est par ce système vasculaire local que l'information hormonale est transmise de l'hypothala-
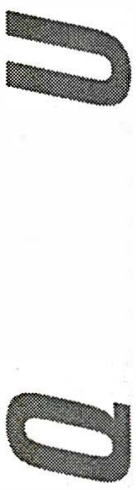
mus à l'adénohypophyse. La destruction du SPHH sans léser la tige pituitaire (expérience réalisée chez le canard grâce à une disposition anatomique particulière) rend l'adénohypophyse non fonctionnelle. On a établi que les neurones hypothalamiques parvocellulaires (noyaux disséminés dans l'hypothalamus médian) libèrent des neurohormones dans le $\mathrm{SPHH}$, qui contrôlent l'activité de l'adénohypophyse. Il existe deux familles de neurohormones : les neurohormones activatrices (releasing hormone) stimulant la libération d'une stimuline adénohypophysaire, ou au contraire inhibitrices (inhibiting hormone), de cette libération. La TRH (thyrotropin releasing hormone), qui stimule la libération de la TSH adénohypophysaire (hormone stimulant l'activité de la thyroïde), est un peptide de 3 acides aminés. La LHRH (luteinizing hormone releasing hormone), 10 acides aminés, stimule la libération de la FSH-LH adénohypophysaire (hormone stimulant l'activité des ovaires et des testicules). La somatostatine, 14 acides aminés, inhibe la libération de l'hormone somatotrope adénohypophysaire (ou hormone de croissance). Le CRF (corticotropin releasing hormone), 41 acides aminés, stimule la libération de l'ACTH adénohypophysaire (hormone stimulant l'activité des glandes corticosurrénales). Peu à peu, toutes les neurohormones hypothalamiques ont été ou seront identifiées. On peut mentionner que, dans le cadre de la recherche des neurohormones contrôlant la libération de la prolactine adénohypophysaire (hormone dont le rôle principal est de stimuler la production du lait au cours de la lactation), on a observé que le facteur inhibiteur de sécrétion n'est pas un peptide, mais une monoamine connue comme neurotransmetteur: la dopamine.

Cette liaison spécifique de chaque neurohormone hypothalamique à une hormone adénohypophysaire est trop schématique. De fait, dans de nombreux cas, certaines interviennent

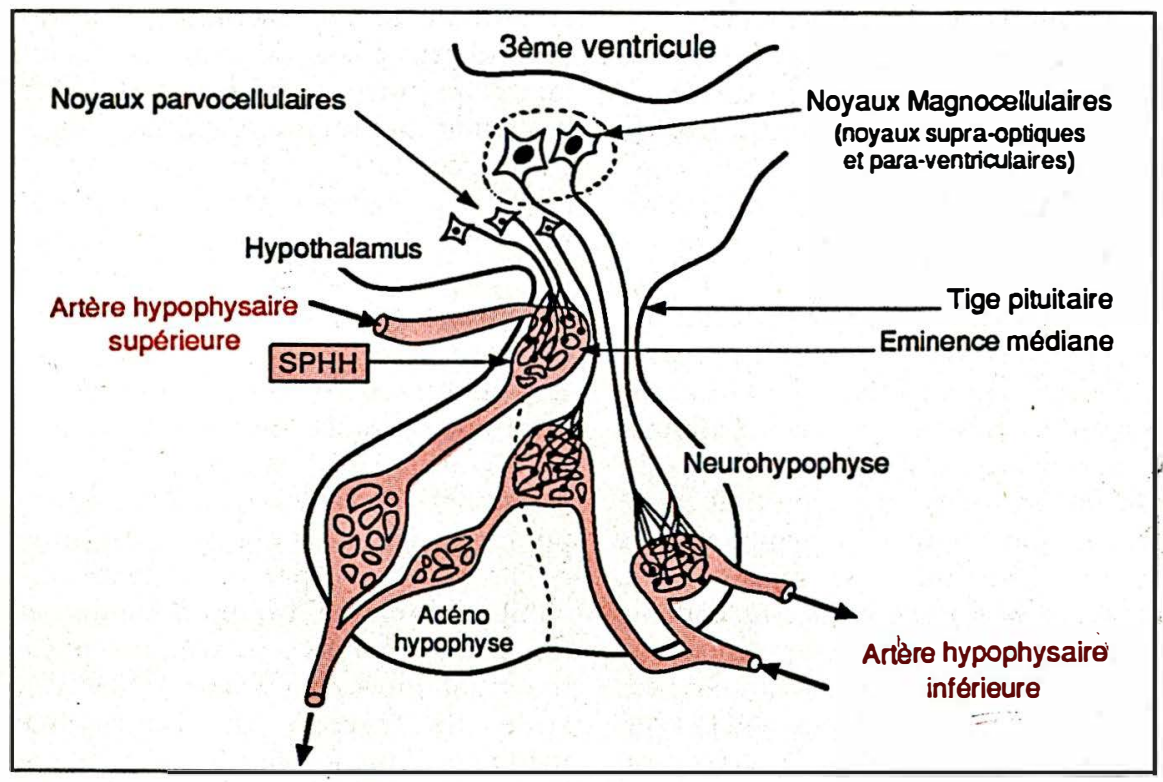

Figure 1. Représentation schématique des relations vasculaires et nerveuses intervenant dans le contrôle neurohormonal exercé par I'hypothala910 dans la régulation de la sécrétion de plusieurs hormones hypophysaires. Ainsi, le TRH stimule non seulement la libération de TSH, mais également celle de la prolactine et de l'hormone de croissance.

Les neurohormones sont stockées au niveau des terminaisons axonales dans des vésicules et leur libération se fait par un processus classique d'exocytose, déclenchée par la survenue, au niveau de la terminaison axonale, d'un potentiel d'action. Dans le cas des systèmes magnocellulaires, cette libération est la conséquence de l'activation par une stimulation sensorielle périphérique d'une boucle sensori-hormonale, par exemple la succion du mamelon mammaire pour la libération d'ocytocine. Dans ce cas particulier, on peut mentionner le fait que les neurones libérant l'ocytocine ont leur organisation anatomo-fonctionnelle complètement bouleversée pendant la période de lactation, et que ces bouleversements sont parfaitement réversibles.

Le développement du concept de neurosécrétion est d'une grande importance car il modifie très profondément la représentation du cerveau véhiculée par la neurotransmission, telle que nous l'avons développée jusqu'à présent dans ce lexique (voir en particulier $m / s \quad n^{\circ} 3$, vol. 5, p. 177), à savoir, comme le propose Claude Debru*, "la représentation d'un réseau de neurones interconnectés, la représentation purement électrique du fonctionnement cérébral comme câblage et champ ". "Les idéalisations du fonctionnement cérébral reposant sur une représentation seulement électrique sont inadéquates. Le cerveau est un ensemble d'organes reliés entre eux non seulement par des fibres nerveuses mais également par voie liquidienne."

Bernard Calvino Jean-Paul Rivot Marc Peschanski

\footnotetext{
- De la neurotransmission à la neuroendocrinologie ", Cahiers d'histoire et de philosophie des sciences, $n^{\circ} 22,1987$.
}

$m / s n^{\circ} 9$, vol. 6 , novembre 90 